Hospitalisation

\section{Readmission of neonates}

\section{R Scott-Jupp}

\section{Commentary on the papers by Oddie et al (see page 119) and Escobar et al (see page 125)}

t wo papers coincidentally submitted to Archives from opposite sides of the Atlantic give an opportunity to point out some interesting similarities and differences in the controversial area of hospital readmission shortly after birth. Clinicians in both the UK and the USA are concerned about the knockon effects of the increasing trend towards earlier neonatal discharge from hospital.

The studies differ significantly in their objectives and methods, so direct numerical comparisons may not be valid. Oddie et al looked at over 11000 births in the Northern NHS region of the UK in 1998, excluding infants less than 35 weeks gestation. ${ }^{1}$ They concentrated on factors associated with early neonatal discharge, and then looked at what influenced readmission to hospital within 28 days. Escobar et al studied a population of over 33000 using the Kaiser Permanente Medical Care Program (KPMCP) in California and Colorado, for which good data are available, in 1998-2000; ${ }^{2}$ they included all gestations and did not look at early discharge at all, but analysed in some detail factors associated with readmission within two weeks. The KPMCP, although not government run, is a managed healthcare system which has been described as being in many ways similar to the British National Health Service. $^{3}$ Its membership is not restricted to the more prosperous sections of society, as may be inferred from the racial distribution of infants in some of the institutions in the study (see table 1 in Escobar et $a l^{2}$ ).

Oddie et al found early discharge to be less likely, not surprisingly, in smaller, lower gestation infants. Social deprivation was associated with earlier discharge; this may reflect economic and family pressures to return home, or possibly a tendency for the more deprived to feel relatively disempowered in negotiating with professionals about the timing of their discharge. Older maternal age was associated with longer stays once other factors were allowed for. Breast feeding led to longer stays, but the association was weak. Their findings on readmission within 28 days are perhaps counter-intuitive to many paediatricians: on multivariate analysis, there was no association between early neonatal discharge and readmission, nor did social deprivation have an influence. Indeed the only significant independent associations they found were breast feeding with reduced readmission rates, and lower gestation with increased rates. They did not study factors such as community support and postnatal visits, but there was considerable variation between the five hospitals in the study. Clinical reasons for readmission were, as expected, a broad range of postnatal problems, probably including a nebulous group of "parental anxiety" admissions with no firm diagnosis; about $9 \%$ were primarily because of jaundice.

Escobar et al looked at a different range of factors influencing readmission in the first two weeks. As in the UK study, lower gestations were more likely to be readmitted (including those that had avoided the neonatal intensive care unit), as were those who were sicker at birth. They did not look at feeding mode, but were able to determine which had received home health visits or had attended outpatients: those who had been visited at home in the first 72 hours after discharge were less likely to be readmitted, unless they had also attended outpatients, in which case readmission was more likely. Presumably this latter category reflects a more vulnerable group. Analysis of race revealed that African-American infants were less likely to be readmitted: if, as is often suggested, race in the USA is taken as a proxy for social deprivation, then this represents an interesting difference from the UK study. Asian infants were more likely to be readmitted, but this was almost entirely explained by jaundice. Again, the diagnoses leading to readmission were as expected, but jaundice was the reason in $34 \%$, much more frequent than in the UK study. This difference is unlikely to be completely explained by the inclusion of more preterm infants in the US study. Management of early neonatal jaundice has been the subject of recent reviews, and clearly practice varies between institutions and between nations. ${ }^{45}$ American practice may be much more cautious in preventing high bilirubin levels. However, as discussed by Escobar et al, home phototherapy has an important role here.

A major difference between the two countries is the statutory provision in the UK of a community based midwifery and health visiting service. These two professions are largely non-existent in the USA. The American study found fewer readmissions for those who received a home visit as part of their KPMCP "package", and this might be analogous to the British postnatal midwife's visit. Unfortunately the UK study had no data on postnatal visits, nor on their communities' policies in this area. It cannot be assumed that postnatal home visits will always reduce readmission rates: where the parent's anxiety exceeds that of the health professional it may do so, but where the parent had been unconcerned and the professional detects a problem, it may have the opposite effect.

A strikingly consistent theme in both studies is the huge variation in readmission rates between neighbouring hospitals. This actually accounts for more of the variation than the "biological" factors already discussed. This is not apparently explained by differences in the degree of social deprivation in the populations served. It is difficult to identify reasons for this: associations with institutional characteristics looked for in the US study were weak and inconsistent, although availability of home phototherapy appears to have some influence. Presumably, in both countries, traditional policies and practices vary between institutions for historical reasons that may have nothing to do with their patient population profile.

From the perspective of a manager or health economist, it might appear that if all institutions could adopt the practices of those with the earliest discharge and lowest readmission rates, then major financial savings could be made. However, we should be cautious about going down this road on the basis of these data, as we know nothing about outcomes, including, possibly, rare but potentially avoidable death and disability; moreover, pressure to send newborns home early, and then to avoid readmission at all costs, may add to the turmoil and anxiety experienced by some vulnerable families that we are unable to quantify.

Arch Dis Child 2005;90:111-112. doi: 10.1136/adc.2004.052563

Correspondence to: $\operatorname{Dr} \mathrm{R}$ Scott-Jupp, Salisbury District Hospital, Salisbury SP2 8BJ, UK; scottjupp@aol.com 


\section{REFERENCES}

1 Oddie SJ, Hammal D, Richmond S, et al. Early discharge and readmission to hospital in the first month of life in the Northern Region of the UK during 1998 a case cohort study. Arch Dis Child 2005:90:119-24.
2 Escobar GJ, Greene JD, Hulac P, et al. Rehospitalisation after birth hospitalisation: patterns among infants of all gestations. Arch Dis Child 2005:90:125-31.

3 Feachem RGA, Sekhri N, White K. Getting more for their dollar: a comparison of the NHS and California's Kaiser Permanente. BM 2002;324:135-43.

\author{
4 Maisels MJ, Watchko JF. Treatment of \\ jaundice in low birthweight infants. \\ Arch Dis Child Fetal Neonatal Ed \\ 2003:88:F459-63. \\ 5 Johnson LH, Bhutani VK, Brown AK. System- \\ based approach to management of neonatal \\ jaundice and prevention of kernicterus. J Pediatr \\ 2002; 140:396-403.
}

Endocrinology

\section{Congenital hypothyroidism}

\section{J H Lazarus}

\section{Commentary on the paper by Oerbeck et al (see page 132)}

$\mathrm{T}$ he introduction of neonatal screening programmes for congenital hypothyroidism in the 1970s is now regarded as a highly cost effective strategy to detect the commonest congenital metabolic disorder seen in the newborn ( 1 in around 4000 births). ${ }^{1}$ There is no doubt that early diagnosis and treatment of the condition has led to the disappearance of mental retardation, which was the most dramatic long term sequel of congenital hypothyroidism. $^{2}$ However, it has been clearly recognised that persistent selective impairments may still occur in these children, such as language delays, minor motor problems, visuospatial defects, and attention problems. ${ }^{3}$ Also, postnatal somatic abnormalities including an accelerated cranial growth ${ }^{4}$ and delayed bone age ${ }^{5}$ to 3 years have been observed, especially in children given high starting doses of levothyroxine. Initially, starting doses of thyroxine were in the range of $8-10 \mu \mathrm{g} / \mathrm{kg} / \mathrm{day},{ }^{6}$ but the dose was later revised upwards to $10-16 \mu \mathrm{g} / \mathrm{kg} / \mathrm{day}^{7}$ There is clearly a need to define the optimal dose of T4 to initiate therapy as well as the desirable levels of serum T4 to be achieved during long term therapy.

This subject was recently discussed by Rovet, ${ }^{8}$ who indicated that a number of studies (for example, BongersSchokking et $a l^{9}$ and Dubuis et $a l^{10}$ ) have shown that a higher dose is beneficial in closing the IQ gap between moderate and severe forms of the disease. However, she has previously recorded a possible increase in neurobehavioural disorder in children who have received higher dose regimens of thyroxine. ${ }^{11}$ Furthermore, hyperthyroxinaemia in rodents has been associated with adverse neurodevelopmental effects. ${ }^{12}{ }^{13}$ What current data are available to substantiate the practice of higher dose initial thyroxine therapy? Distinction must be made between the initial serum
$\mathrm{T} 4$ at diagnosis, the starting dose of T4, and the maintenance serum levels of thyroid hormones in the outcome assessment. Two recent studies emphasise the importance of the initial serum T4. Ng and colleagues ${ }^{14}$ found that the initial T4 was an independent factor (inversely related) in the control of head growth in the first three years in 125 subjects with congenital hypothyroidism $(\mathrm{CH})$. In $31 \mathrm{CH}$ subjects studied at 4 years of age, a higher baseline T4 was one of the main predictors of increased verbal IQ but, interestingly, levothyroxine dose at the beginning of treatment and thyroid hormone levels during treatment did not relate to IQ outcome. ${ }^{15}$ In relation to the initial starting dose of T4, Gauchard and colleagues ${ }^{16}$ showed in 17 patients that early normalisation of TSH (before 3 months) was necessary to allow for normal neurosensorial afferent pathway development (vestibular, proprioceptive), as well as pathways of central integration (cerebellum, vestibular nuclei). The Norwegian study of 49 patients ${ }^{17}$ showed that the initial T4 dose predicted verbal IQ at age 20, but the authors have expressed concern at some negative associations between high dose treatment and developmental outcome. ${ }^{18}$ Nevertheless, a recent Cuban study of $100 \mathrm{CH}$ children studied at 8.2 years showed that total IQ was related to the initial T4 dose. ${ }^{19}$ A careful study by Simoneau-Roy and colleagues ${ }^{20}$ showed that children with severe $\mathrm{CH}$ treated early with a high dose (median $12 \mu \mathrm{g} / \mathrm{kg} /$ day) of levothyroxine had normal global development and behaviour at school entry. However, as these authors noted, the number of subjects was small, indicating the need for further studies. The Norwegian workers have now extended their study of the 49 subjects referred to above in an attempt to describe the psychological problems in these young adults and to evaluate any negative effects of high dose thyroxine replacement therapy. ${ }^{21}$ The results indicate that the $\mathrm{CH}$ group, perhaps not surprisingly, had lower performance levels than their sibling controls for some aspects of memory, and attention, and had more behaviour problems. Importantly, a high T4 starting dose $(\geqslant 7.8 \mu \mathrm{g} / \mathrm{kg} /$ day $)$ had no adverse effect on outcome at age 20 . Furthermore, there was no deleterious effect of higher T4 levels during infancy, early childhood, or at assessment on the higher order cognitive skills. This study, which divided the initial treatment T4 dose into two groups similar to the Toronto workers, ${ }^{8}$ adds significant evidence that a high starting dose of T4 in $\mathrm{CH}$ is not harmful in the long term and may be beneficial in some outcome measures.

There are other physiological factors to be considered in relation to thyroxine delivery to developing neural tissue. The fetus with $\mathrm{CH}$ derives its $\mathrm{T} 4$ mostly from the mother through gestation. Details of the transplacental passage of the hormone are certainly incomplete, but the role of the deiodinase enzymes, particularly type 3, in acting as "a gatekeeper" in this process is under intensive study. ${ }^{22}$ Variations in the rate of different areas of brain development during gestation and their detailed response to $\mathrm{T} 4$ in marginally hormone deficient situations are further factors which cause abnormal brain morphology at birth. ${ }^{23}{ }^{24}$ In addition, the complexity of thyroid hormone action in the developing brain is compounded by temporal and regional variations in metabolism, receptor and gene expression. ${ }^{25}$ On a practical level the maternal thyroid hormone supply to the fetus is dependent on the iodine supply which may be precarious. ${ }^{26}$

It is probable that the classification of $\mathrm{CH}$ into mild moderate and severe, for example, is too simplistic in relation to brain architecture and may account for variation in outcome in children with similar initial hormone levels. Hence it is not surprising that studies in children using psychological outcomes will have differing specificities and sensitivities. From the available clinical evidence, to which the study of Oerbeck et al has contributed significantly, it would appear that the starting dose of levothyroxine should be higher rather 
than lower, and that the long term risks of adverse psychological performance are small. A large scale multicentre study is indicated to clarify the concerns of the "low dose" group of investigators. Meanwhile advances in our understanding of thyroid hormone action on the developing brain will improve our ability to make these important clinical judgements.

Arch Dis Child 2005;90:112-113.

doi: 10.1136/adc.2004.061572

Correspondence to: Prof. J H Lazarus, Professor of Clinical Endocrinology, University of Wales College of Medicine, Cardiff CF14 4XN, UK; Lazarus@cf.ac.uk

\section{REFERENCES}

1 Pass K. Overview of newborn screening for congenital hypothyroidism. In: Hauser P, Rovet J, eds. Thyroid diseases of infancy and childhood. Washington, DC: American Psychiatric Press, 1999:59-84

2 New England Congenital Hypothyroidism Collaborative. Effects of neonatal screening for hypothyroidism: prevention of mental retardation by treatment before clinical manifestations. Lancet $1981 ; 2: 1095-8$

3 Rovet J. Behavioral and cognitive abnormalities associated with congenital hypothyroidism. In: Hauser P, Rovet J, eds. Thyroid diseases of infancy and childhood. Washington, DC: American Psychiatric Press, 1999:85-126.

4 Aronson R, Ehrlich RM, Bailiey JD, et al. Growth in children with congenital hypothyroidism detected by neonatal screening. J Pediatr 1990;116:33-7.

5 Salerno M, Micillo M, Di Maio S, et al. Longitudinal growth, sexual maturation and final height in patients with congenital hypothyroidism detected by neonatal screening. Eur J Endocrinol 2001; 145:377-83

6 Fish LH, Schwartz HL, Cavanaugh J, et al. Replacement dose, metabolism and biovavailability of levothyroxine in the treatment of hypothyroidism. Role of triiodothyronine in pituitary feedback in humans. N Engl J Med 1987:316:764-70

7 Americal Academy of Pediatrics, Section on Endocrinology and Committee on Genetics. Newborn screening for congenital hypothyroidism: recommended guidelines. Pediatrics 1993:91:1203-9.

8 Rovet JF. In search of the optimal therapy for congenital hypothyroidism. J Pediatr 2004; 144:698-700.

9 Bongers-Schokking JJ, Koot HM, Wiersma D, et al. Influence of timing and dose of thyroid hormone replacement on development in infants with congenital hypothyroidism. J Pediatr 2000;136:292-7.

10 Dubuis JM, Gloriveux J, Richer F, et al. Outcome of severe congenital hypothyroidism: closing the developmental gap with early high dose of levothyroxine treatment. J Clin Endocrinol Metab 1996:81:222-7.

11 Rovet JF, Ehrlich RM. Long-term effects of Lthyroxine therapy for congenital hypothyroidism. $J$ Pediatr 1995; 126:380-6

12 Davenport JW, Gonzalez LM. Neonatal thyroxine stimulation in rats: accelerated behavioral maturation and subsequent learning deficit. J Comp Physiol 1973;54:397-408

13 Stone JM, Greenough WT. Excess neonatal thyroxine effects on learning in infant adolescent rats. Dev Psychobiol 1975;8:469-88.

$14 \mathrm{Ng} \mathrm{SM}$, Wong SC, Didi M. Head circumference and linear growth during the first 3 years in treated congenital hypothyroidism in relation to aetiology and initial biochemical severity. Clin Endocrinol 2004:61:155-9.

15 Kreisner E, Schermann L, Camargo-Neto E, et al. Predictors of intellectual outcome in a cohort of Brazilian children with congenital

hypothyroidism. Clin Endocrinol 2004:60:250-5.

16 Gauchard GC, Deviterne D, Leheup B, et al. Effect of age at thyroid stimulating hormone normalization on postural control in children with congenital hypothyroidism. Dev Med Child Neurol 2004:46:107-13.

17 Orbeck B, Sundet K, Kase BF, et al. Congenital hypothyroidism: influence of disease severity and L-thyroxine treatment on intellectual, motor, and school-associated outcomes in young adults. Pediatrics 2003;112:923-30.

18 Heyerdahl S, Oerbeck B. Congenital hypothyroidism: developmental outcome in relation to levothyroxine treatment variables. Thyroid 2003;13:1029-38

19 Alvarez Gonzalez MA Carvajal Martinez F, Perez Gesen C, et al. Prognosis of cognition in congenital hypothyroidism following early treatment. Double effect hypothesis. Rev Neurol 2004;38:513-17.

20 Simoneau-Roy J, Marti S, Deal C, et al. Cognition and behavior at school entry in children with congenital hypothyroidism treated early with high-dose levothyroxine. J Pediatr 2004; 144:698-700.

21 Oerbeck B, Sundet KS, Kase BF, et al. Congenital hypothyroidism: no adverse effects of high dose thyroxine treatment on adult memory, attention, and behaviour. Arch Dis Child 2005:90:132-7.

22 Kohrle J. The deiodinase family: selenoenzymes regulating thyroid hormone availability and action. Cell Mol Life Sci 2000;57:1853-63

23 Lavado-Autric R, Auso E, Garcia-Velasco JV et al. Early maternal hypothyroxinemia alters histogenesis and cerebral cortex cytoarchitecture of the progeny. J Clin Invest 2003; 111:1073-82.

24 Kester MH, Martinez de Mena R, Obregon MJ, et al. lodothyronine levels in the human developing brain: major regulatory roles of iodothyronine deiodinases in different areas. J Clin Endocrinol Metab 2004;89:3117-28.

25 Anderson GW, Schoonover CM, Jones SA. Control of thyroid hormone action in the developing rat brain. Thyroid 2003;13:1039-56.

26 Pearce EN, Bazrafshan HR, He X, et al. Dietary iodine in pregnant women from the Boston, Massachusetts area. Thyroid 2004; 14:327-8.

doctors and nurses pass their lives with children, talk to none but children, touch none but children and comfort and cure none but children.

'Is there really such a place?' asked the old woman with a gaze of wonder."

Dickens later describes how the child was permitted the comfort of bringing favourite toys to the hospital:

"At the Children's Hospital, the gallant steed, the Noah's Ark, the yellow bird and the officer in the Guards were made as welcome as their child owner."

The early ancestors of the modern paediatric hospital (now, in many cases, wisely renamed health centres) included the foundling hospitals, best known for interminable stays, high mortality, and for being the sites where psychosocial deprivation, or "failure to thrive" was first identified as a clinical entity. The paediatric hospital's ancestry also includes isolation hospitals for
"We want to move Johnny to a place where there are none but children; a place set up on purpose for sick children; where the good monastic unicellular roots. In health care, as in life, old traditions die hard.
The mere idea of a hospital dedicated exclusively to the care of children is a 19th century, Charles Dickens was a igorous campaigner for the support for The Hospital for Sick Children, Great published in 868 , he provided one how Mrs Boffin persuades an elderly hospitals now encourage the support of woman to seek good care for an ill child: and friends as an aid to recovery. But in too many adult hospitals, the 
contagious disease ("fever hospitals") and children's orthopaedic hospitals often known, depressingly as ("hospitals for crippled children").

Paediatrics has been a leader in fostering entirely new kinds of structural and functional health care facilities-places where families are not merely welcome, but play an increasing role as "care partners" in helping children recover. Hearing a child crying in a modern paediatric hospital is now the exception. Not so many years ago, it was the rule.

Hand in hand with the dramatic evolution of physical environments, and of our attitudes towards children's family members (remember when they were referred to as "visitors"?) has been a remarkable trend towards minimising the number and duration of a paediatric hospital admissions without prejudicing children's health and rate of recoveryoften with the conviction that children's health is better served through alternative approaches to paediatric care.

There are innumerable specific examples of this revolution in paediatric health care, including day surgery, home care, palliative care, short stay (observation) units, and most recently, tele-medicine to serve children living in remote areas.

A live tele-medicine consultation service has recently been shown to reduce dramatically the need to transfer critical care patients from a rural intensive care unit to a highly specialised central paediatric intensive care facility. ${ }^{2}$

In another innovative study of alternatives to traditional care, heart sounds from 87 patients with and without murmurs in distant rural areas of Norway were recorded with a sensor based stethoscope, e-mailed to a remote computer, and randomly distributed to four cardiologists, who had to categorise them as "no murmur", "innocent murmur", or "pathological murmur". The cardiologists spent an average of 2.1 minutes per case. Mean sensitivity and specificity were $89.7 \%$ and $98.2 \%$ respectively, with low inter- and intraobserver variability. ${ }^{3}$ In a commentary that accompanied this report, Wren ${ }^{4}$ goes a step further, suggesting that the paediatric cardiologist him/herself could be replaced by a computer, citing a recent report showing $100 \%$ sensitivity and specificity of an artificial neural network in assessment of murmurs, a track record better than the average paediatric cardiologist!

If such innovations have achieved nothing else, they have certainly reduced dramatically the duration of paediatric hospital admissions, and many of us are convinced that, as a result, quality of care, speed of recovery, parent satisfaction, and other desirable objectives are being met. But if challenged, can we back up such fervent convictions with solid evidence?

In this issue, Dr David Ogilvie tackles this question head on. ${ }^{5}$ He challenges us to produce the evidence that hospital based alternatives to paediatric admission do more good than harm, and reports the findings of his systematic review of interventions designed to provide alternatives to acute admission in medical paediatrics. His search of the literature has been thorough, his inclusion criteria rigorous, and his evaluation evidence based. It is interesting to note that he unearthed only a single randomised controlled trial, and even this one was not without methodological blemishes. But despite such shortcomings, certain consistent themes emerge from Ogilvie's analysis. For one thing, parent satisfaction levels with such alternatives to traditional care have been consistently high. Parents as a group are rarely wrong in their judgements, and one might question whether levels of parent satisfaction should be regarded as a subjective or an objective finding. The distinction may be rather arbitrary. An opinion, after all, is a kind of "fact".

Dr Ogilvie puts us on notice to adopt more rigorous methods to evaluate any and all potential improvements in delivery of paediatric health care. He correctly advises us to perform our measurements using bi-directional scales, so that both positive and negative outcomes are assessed. There are, after all, few forms of treatment that do not include both positive and negative effects. It is our responsibility to establish for any health care innovation, that good outweighs harm by a wide, statistically significant margin.

Arch Dis Child 2005;90:113-114.

doi: 10.1136/adc.2004.057075

Correspondence to: $\operatorname{Dr}$ R B Goldbloom, Dalhousie University, Halifax, Nova Scotia, Canada; richard.goldbloom@iwk.nshealth.ca

\section{REFERENCES}
1 Dickens C. Our Mutual Friend. London, 1858 2 Marcin JP, Nesbitt TS, Kallas HJ, et al. Use of telemedicine to provide pediatric critical care inpatient consultations to underserved rural northern California. J Pediatr 2004; 144:375-80.
3 Dahl LB, Hasvold P, Arild E, et al. Heart murmurs recorded by a sensor based electronic stethoscope and e-mailed for remote assessment Arch Dis Child 2002;87:297-301.
4 Wren C. Technological advances and assessment of children with murmurs. Arch Dis Child 2002;87:301-2.
5 Ogilvie D. Hospital based alternatives to acute paediatric admission: a systematic review. Arch Dis Child 2005;90:138-42.

the fact that the data of Dr Lee et al are based on pregnancies occurring only in the United Kingdom, the findings verify two of the most important findings in our longitudinal, prospective study, which collected data from three different countries: the United States, Canada, and Germany. In any international study such as the latter, cultural and social differences can always interfere with statistical analyses. Therefore the fact that both studies support and concur that pregnancies in control by the first 10 weeks of pregnancy resulted in normal intellectual development in the offspring at 6-8 years is important information. Furthermore, both studies observed that the occurrence of congenital heart disease in the offspring of women in good metabolic control very

early in pregnancy was reduced to $1-2 \%$,

which support the main findings of the Collaborative Maternal PKU Study sponsored by the National Institute of Child Health and Human Development in Bethesda, Maryland, USA. ${ }^{2}$ Despite women with phenylketonuria (PKU). ${ }^{1}$
This paper provides important data 
compared to $14-17 \%$ in those not in good control.

It is true that the numbers in the British study are smaller than those in the Collaborative Study and alone would not satisfy strong biostatistical evaluation. Only 32 offspring had IQ data at 8 years, whereas in the latter study, follow up data at 6-8 years of age were examined in 231 children.

It is also true that the two studies were organised in a different fashion and yet arrived at similar conclusions. The British study was based on a registry, which was analysed retrospectively for significant outcomes, whereas the Collaborative Study was longitudinal in nature and based on a specific protocol. It is remarkable that the results coincide on the major outcome data.

In reviewing both published studies, it is clear that a public health effort will have to be mounted to further improve outcome. For example, only some 50\% of the women were treated before conception. While we currently look on this as significant progress, future studies will undoubtedly show more improvement as we begin to deal primarily with women of normal intelligence. The two reports involve a significant number of women with IQs of 85 or below. For discussion purposes, let us assume that the IQs in the British mothers were similar to those of mothers in the Collaborative Study. If so, $51 \%$ of the women with PKU reported in the Collaborative Study had documented IQ assessments of less than 85 on the Wechsler Adult Intelligence Scale. This significant reduction in maternal IQ in both studies is undoubtedly related to the diet discontinuation phase of treatment occurring in many countries during the period between 1965 and 1985. Today diet continuation for women with phenylketonuria is accepted procedure for most countries. Therefore future studies hopefully will be based on outcome in women with normal intelligence. It is my opinion that the availability of mutational data will aid us in selecting women with PKU who definitely must remain on a phenylalanine restricted diet, especially during their childbearing years.

The future use of tetrahydrobiopterin (BH4) in the treatment of pregnancies either alone or in combination with phenylalanine restriction in the mother's diet also offers hope for improved fetal outcome. The IQ spread in the mothers evaluated in the Collaborative Study was 58-130. There were 48 women in the Collaborative Study who had mild hyperphenylalaninaemia. In the evaluation of the offspring of these 48 women, 40 of whom were untreated and eight of whom were treated with a phenylalanine restricted diet, Levy et al reported that the mean IQ of the 6-8 year old offspring was $102 .^{3}$ No significant difference in IQ was found between the offspring of the women who were untreated and those who were treated. Future studies may document that treating women with PKU hopefully will produce a better fetal outcome in these pregnancies.

In conclusion, it is for future investigators to verify these new treatment modalities, and perhaps $\mathrm{BH} 4$ will prove useful in improving intellectual outcome even in women with classical PKU with two severe mutation of the phenylalanine hydroxylase gene.

Arch Dis Child 2005;90:114-115.

doi: 10.1136/adc.2004.054346

Correspondence to: Dr R Koch, Principal Investigator, Maternal PKU Collaborative Study, Los Angeles, California, USA; rkoch8@ earthlink.net

\section{REFERENCES}

1 Lee PJ, Ridout D, Walter JH, et al. Maternal phenylketonuria: report from the United Kingdom Registry 1978-97. Arch Dis Child 2005:90:143-6.

2 National Institute of Child Health and Human Development. The Maternal Phenylketonuria Collaborative Study: new developments and the need for new strategies. Pediatrics 2003;112:1513-87.

3 Levy HL, Waisbren SE, Guttler F, et al. Pregnancy experiences in the woman with mild hyperphenylalaninemia. Pediatrics 2003;113(6 pt 2):1548-52. 